\title{
Acute Myelopathy or Cauda Equina Syndrome in HIV-Positive Adults in a Tuberculosis Endemic Setting: MRI, Clinical, and Pathologic Findings
}

\author{
S. Candy, G. Chang, and S. Andronikou
}

\begin{abstract}
BACKGROUND AND PURPOSE: Cape Town is the center of an HIV-tuberculosis coepidemic. This study's aim was to highlight the importance and to describe the MR imaging features of tuberculosis in acute myelopathy and cauda equina syndrome in HIV-positive adults. To accomplish this we retrospectively reviewed the MR imaging and clinico-pathologic findings of HIV-positive patients presenting to our hospital with recent onset paraplegia and sphincter dysfunction over a 4-year period, 2008-2011.
\end{abstract}

MATERIALS \& METHODS: MR imaging, CD4 count, and CSF analysis and pathology were correlated in 216 cases.

RESULTS: Fifty-eight percent (127) of subjects were female. The mean age was 37 years. The median CD4 count was 185 cells/ $\mu$ L. Twenty-five percent (54) of patients were on antiretroviral therapy. MR imaging showed spondylitis in 30\% (65). The median CD4 count in these patients was significantly higher than in the remainder. Disk destruction was common and $10 \%$ had synchronous spondylitis elsewhere in the spinal column. Thirty percent (64) had features of myelitis/arachnoiditis. Twenty-five percent (55) had no MR imaging abnormality. In 123 (57\%) of cases with a definitive etiology on CSF culture or biopsy, 84 (68\%) were attributable to tuberculosis including all spondylitis cases and $40 \%$ of nonspondylitis cases. Twelve (10\%) were due to nontuberculous infection and $12(10 \%)$ had HIV-associated tumors including 2 rare Epstein-Barr-related tumors.

CONCLUSIONS: In our setting, acute onset myelopathy/cauda equina syndrome in HIV-positive patients is largely attributable to tuberculosis with nonspondylitic forms being more common than spondylitis and associated with a lower CD4 count.

ABBREVIATIONS: $\mathrm{TB}=$ tuberculosis; $\mathrm{ARV}=$ antiretroviral; $\mathrm{EB}=$ Epstein-Barr virus

$\mathrm{C}$ ape Town, South Africa is presently experiencing a coepidemic of HIV and tuberculosis (TB). South Africa currently ranks fourth in the world for tuberculosis disease with an annual incidence rate in the Western Cape of 935/100,000. ${ }^{1}$ The HIV epidemic has greatly increased the prevalence of both drug sensitive and resistant forms of tuberculosis and extrapulmonary forms of the disease. ${ }^{2}$ Spinal tuberculosis particularly is reported to be more common in persons infected with HIV. $^{3}$

Over the past decade, increasing numbers of young HIVpositive adults have been referred to our institution for spinal

Received November 11, 2013; accepted after review January 15, 2014.

From the Department of Radiology (S.C., G.C.), Groote Schuur Hospital, Faculty of Health Sciences, University of Cape Town, Cape Town, South Africa; and Department of Radiology (S.A.), Faculty of Health Sciences, University of the Witwatersrand, Johannesburg, South Africa.

Please address correspondence to Sally Candy, MD, Department of Radiology, Groote Schuur Hospital, Faculty of Health Sciences, University of Cape Town, Anzio Rd, Observatory, Cape Town, South Africa 7925.

http://dx.doi.org/10.3174/ajnr.A3958
MR imaging after the onset of myelopathy or cauda equina syndrome. Although many of these patients have the typical MR findings of TB spondylitis, many do not. Nonspondylytic spinal tuberculosis has not received wide attention in the literature and in particular has not been widely reported in association with HIV.

\section{Aim}

This study's aim was to highlight the importance of tuberculosis and document the MR imaging findings in relation to other causes in the setting of acute myelopathy and cauda equina syndrome in HIV-positive adults.

\section{METHOD \\ Study Design}

The design was a retrospective descriptive study of MR imaging scans and clinical/laboratory data in HIV-positive patients presenting with paraplegia and sphincter dysfunction to 1 tertiary referral center in the Western Cape of South Africa. 


\section{Defining the Study Population}

The study population included patients who had received MR imaging scans for recent onset myelopathic or cauda equina symptoms at our institution, which provides MR imaging, specialist neurologic, neurosurgical, and orthopaedic care to the population of Greater Cape Town (population 3 million).

\section{MR Imaging Scanning}

All imaging was performed on the same 1.5T MR scanner (Symphony; Siemens, Erlangen, Germany). A routine protocol was observed: T2 sagittal whole spine and T2 axial (through any area of pathology) with T1 sagittal and axial images in selected cases. FISP imaging of the chest and abdomen was used to determine the presence of lymphadenopathy, solid organ lesions, and marrow edema. In accordance with hospital protocol in a resource constrained environment, gadolinium was administered only when an intramedullary lesion, epidural abscess, or arachnoiditis was suspected $(42 \%$ of those without spondylitis received gadolinium). Diffusion-weighted spinal imaging was not successfully implemented at the time of the study.

\section{Source of Data, Inclusion, and Exclusion Criteria}

A manual search of the MR imaging archive was conducted for referrals of recent onset myelopathic or cauda equina symptoms (lower limb weakness with loss of sphincter control with or without a sensory level), in HIV-positive adults over a 42-month period (2008-2011). The hospital records and National Health Laboratory Service data base were reviewed to confirm HIV and antiretroviral (ARV) status, a diagnosis of pre-existing tuberculosis and antituberculous treatment, age, sex, CD4 count, CSF microscopy, biochemistry, and culture yield from any site. Patients were excluded when HIV status was not confirmed.

\section{Determining the Diagnosis}

For the purpose of the study, etiology was confirmed by using laboratory information. The diagnosis of tuberculosis was made definitively when culture was positive or when acid fast bacilli were identified on a Ziell Nielsen stain from any tissue sample (including samples from sites outside the CNS).

An alternative diagnosis was made where an organism other than Mycobacterium tuberculosis was observed directly or on CSF culture. Neoplasms were diagnosed on fine-needle aspiration or tissue biopsy.

\section{MR Imaging Diagnoses}

The MR imaging scans were evaluated by 1 senior neuroradiologist for the presence of pathologic features in the spinal column, spinal cord, thecal sac, and outside of the CNS. These findings were used to define the following diagnostic imaging categories: spondylitis, myelitis/arachnoiditis, bone neoplasm, nonbone neoplasm, isolated syrinx, and other (Appendix 1).

\section{Assessment and Analysis}

1. The causes of acute paraplegia and the extent of spondylitis and nonspondylitic lesions were determined.

2. The CD4 count was compared in those with and without spondylitis on MR imaging.
3. The CD4 counts were compared for those on ARVs with those who were not.

4. The MR imaging appearance, CSF, and biopsy findings in all patients are described with particular reference to the imaging findings in those patients with a definitive diagnosis of tuberculosis.

5. The subgroup of patients with a definitive etiologic diagnosis (TB or alternative causation) was analyzed to determine the causal relationship of TB with myelopathy/cauda equina.

6. Statistical analysis was done by using the Mann Whitney $U$ test to assess differences between groups.

\section{Limitations}

The retrospective nature of the study meant that no control was possible on standardizing laboratory tests to confirm an etiologic diagnosis. MR imaging scanning techniques were limited to standard hospital protocol and gadolinium was not routinely used.

\section{RESULTS}

\section{Demographics}

From a group of 227 patients, 11 were excluded because HIV was not confirmed or the clinical criteria of paraplegia and loss of sphincter function were not met. The study group (216 cases) comprised 127 women (58\%) and 89 men (42\%). The mean age was 36.7 years (range 19-64 years; men 39 years, women 35 years). The median CD4 count was 185 cells/ $\mu$ L. Fifty-four (25\%) patients were on ARVs, though the duration of their treatment and their pretreatment CD4 count were not available. Fifty-five patients were being treated for documented tuberculosis, though only 9 had documented tuberculous meningitis at the time of onset of myelopathic/cauda equina symptoms.

\section{Overall Analysis of MR Imaging Findings for the Total Group ( $n=216)$}

Fifty-five (25\%) of the patients had no abnormality on MR imaging, 65 (30\%) had spondylitis, 64 (30\%) had myelitis/arachnoiditis/intramedullary lesions. The remaining $15 \%$ comprised 10 cases of lymphoma, 2 Epstein-Barr (EB)-related tumors, 7 bone tumors, 8 disk herniations, 2 cases of syrinx, and 3 cases in which the findings could not be categorized (Table land Appendix 2).

\section{CD4 Counts and MR Imaging Findings}

CD4 Counts in Patients on ARVs (54) versus Those Not on ARVs (162). There was no difference in the mean CD4 count between those on ARVs and those who were not ( 185 cells $/ \mu \mathrm{L}$ in each group).

No significant differences were found in either the CSF or on MRI between those on ARVs versus those not on ARVs: Spondylitis in $35 \%$ on ARVs versus $28 \%$ in those not; myelitis $(33 \%$ versus $28 \%$ ); no MR abnormality (20\% versus $27 \%$ ); lymphoma (6\% in each group); incidental findings (6\% versus 10\%).

CD4 Counts in Patients with Spondylitis (65) versus Those without Spondylitis (161). The median CD4 count for the entire group was 185 cells $/ \mu \mathrm{L}$. We found a significant difference in the median CD4 counts of patients with spondylitis ( 225 cells/ $\mu \mathrm{L})$ and those without spondylitis ( 147 cells $/ \mu \mathrm{L}$ ) by using the Mann-Whitney $U$ test $(P=.0017)$. 
Table 1: MRI-based pathologic categories in 216 patients with HIV and acute onset myelopathy/cauda equina

\begin{tabular}{|c|c|c|c|c|c|c|c|c|c|c|}
\hline MRI Normal & \multicolumn{10}{|c|}{ MRI Abnormal } \\
\hline \multirow[t]{3}{*}{$\begin{array}{c}\text { Normal } \\
\text { Group }\end{array}$} & $\begin{array}{l}\text { Spondylitis } \\
\text { Group }\end{array}$ & \multicolumn{9}{|c|}{ Nonspondylitis Groups } \\
\hline & \multirow[b]{2}{*}{ Spondylitis } & \multirow{2}{*}{$\begin{array}{c}\text { Arachnoiditis-Myelitis } \\
\text { Intramedullary } \\
\text { Tuberculomas }\end{array}$} & \multicolumn{2}{|c|}{ Neoplasm Nonbone } & \multicolumn{3}{|c|}{ Neoplasm Bone } & \multicolumn{3}{|c|}{ Other } \\
\hline & & & HIV Associated & Lymphoma & Lymphoma & Plasmacytoma & Mets & Disk & Syrinx & $?$ \\
\hline 55 & 65 & 64 & 2 & 10 & & 3 & 4 & 8 & 2 & 3 \\
\hline
\end{tabular}

Note:-? indicates no final diagnosis

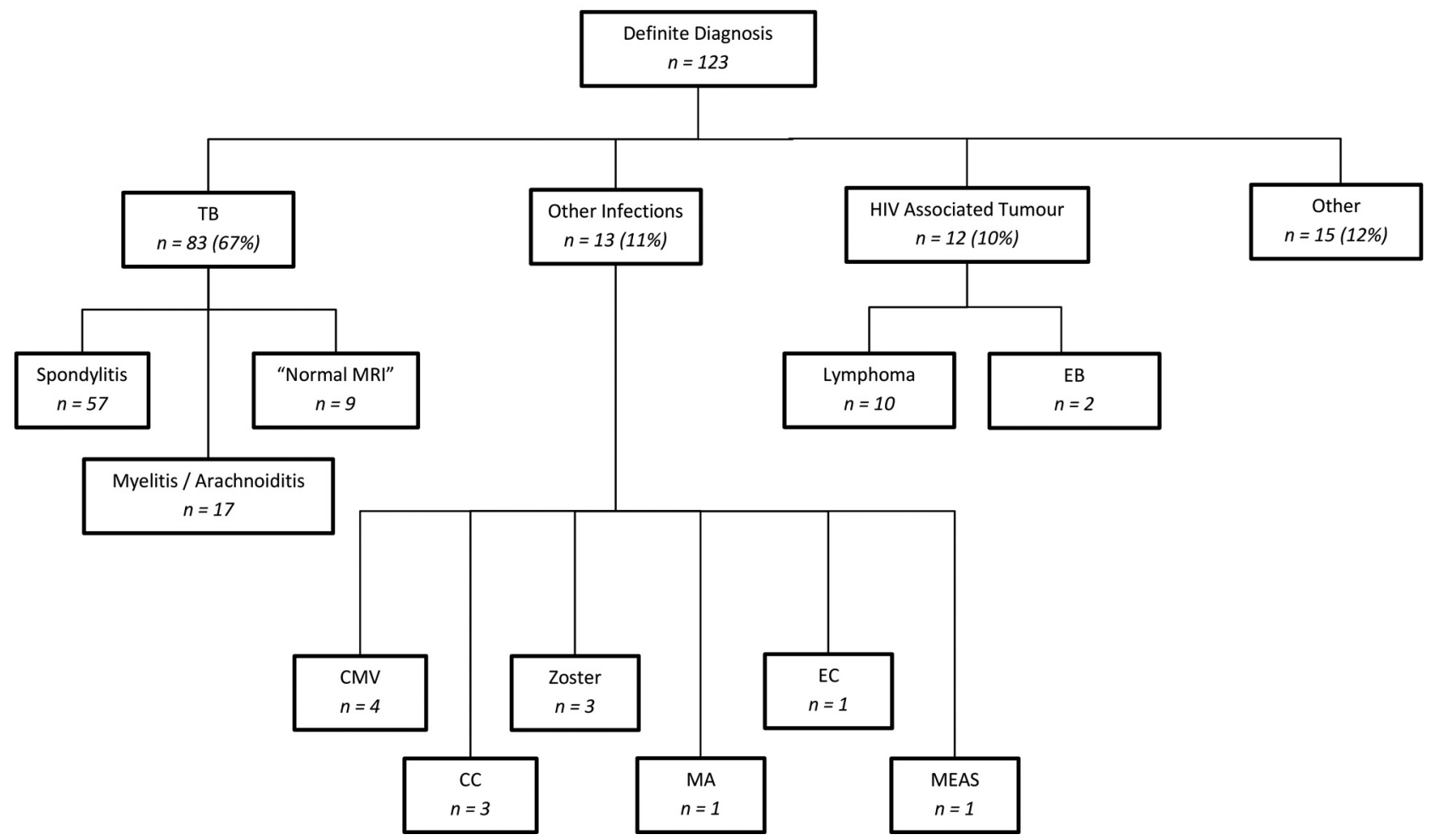

FIG 1. Summary of causation of myelopathy. Abbreviations: CC indicates cryptococcus; Zoster, Varicella zoster; MA, Mycobacterium avium; $\mathrm{EC}$, enterococcus; and MEAS, measles; CMV, cytomegalovirus.

\section{Clinical and Laboratory Diagnosis}

Patients with a Definitive Etiologic Diagnosis $(n=123)$. A definitive etiologic diagnosis was made in 123 of 216 (57\%) (Fig 1). Most of these cases (84) (68\%) were attributable to TB, including 1 case of Mycobacterium avium. Multidrug resistance was found in only 1 of the 84 tuberculosis cases. Thirteen (10\%) patients had nontuberculous infections. Twelve (10\%) had HIV-associated tumors, of which 10 were lymphoma (6 Burkitt, 2 large B-cell, and 2 plasmablastic variant), and 2 were EB-related tumors ( 1 angiomyofibroma and 1 myopericytoma). Fifteen patients (12\%) had non-HIV-related conditions ( 3 plasmacytoma, 4 metastases, and 8 disk herniations). There were no documented cases of bacterial or fungal infection.

Of the 84 cases of culture-proved Mycobacterium tuberculosis, 57 (68\%) presented with MR imaging features of spondylitis, 17 (20\%) with myelitis, and 10 (12\%) had no discernible MR imaging abnormality. The case of Mycobacterium avium had normal MR imaging. Of the 12 with nontuberculous infection, 4 had cytomegalovirus ( 2 with MR imagingdiagnosed myelitis and 2 with no MR imaging abnormality).
Table 2: Mycobacterium tuberculosis as a cause of myelopathy/ cauda equina syndrome in subset of patients with a confirmed aetiology on laboratory testing

\begin{tabular}{cccc}
\hline & MRI Spondylitis & MRI Nonspondylitis & Total \\
\hline TB & & & \\
Yes & 57 & 26 & 83 \\
No & 0 & 40 & 40 \\
Total & 57 & 66 & 123 \\
\hline
\end{tabular}

There was 1 case with both TB and Cryptococcus that had florid arachnoiditis and myelitis. There were 2 with Cryptococcus that had normal MR imaging. There were 3 cases of Varicella zoster, 1 of Enterococcus, and 1 of measles that presented with myelitis.

\section{Causal Relationship of TB with Myelopathy/Cauda Equina}

Of the total number of spondylytic cases seen on MR imaging, $88 \%(57 / 65)$ had a confirmed laboratory diagnosis and all of these were attributable to TB (Table 2). In addition, of all cases with a confirmed laboratory diagnosis that did not have spondylitits, $40 \%(26 / 65)$ were attributable to TB. 


\section{Comparison of Patients with Myelitis/Arachnoiditis with Those with a Normal MR Imaging with Regard to CSF Parameters}

Using the Mann-Whitney statistical test, there was a significant difference between the median CSF lymphocyte count $(P=$ $.0053)$ and protein levels $(P=.0017)$ in those patients with myelitis/arachnoiditis on MR imaging compared with those with no abnormality on MR imaging. The median polymorphonuclear count was not significantly different $(P=.2082)$ (Table 3 ).

\section{Pathologic Features on MR Imaging}

MR Imaging Features in the Spondylitis Group: $(n=65)$. The average number of contiguous affected bodies was 3 (range 1-9). The spinal location was cervical in $20 \%$ (13/65), thoracic in $62 \%$ (40/65), and lumbar/sacral 18\% (12/65).

Disk abnormality (destruction) was present in $40 / 65$ cases (62\%). Epidural cord or thecal sac compression was present in all but 1 patient (98\%). Discontinuous synchronous osteitic lesions were found in $7 / 65(10 \%)$. Psoas and/or paravertebral abscess collections accompanied spondylitis in 48/65 (76\%) (Fig 2).

\section{MR Imaging Features in the Myelitis/Arachnoiditis Group $(n=64)$}

Cord Edema. Fifty-three of the 64 patients (83\%) had high signal in the cord on T2WI. Involvement of the entire cord from craniocervical junction to conus was documented in $8 / 53$ (15\%). In addition, 26/53(49\%) had cervico-thoracic involvement, 5/53 (9\%) had signal abnormality only in the cervical region, and 22

Table 3: Comparison of myelitis group and normal MRI groups with regard to CSF parameters using Mann-Whitney $U$ test

\begin{tabular}{|c|c|c|c|}
\hline MRI Group & $\begin{array}{l}\text { Lymphocyte } \\
\text { Count Median } \\
\left(\text { per } \mathrm{mm}^{3} \text { ) and }\right. \\
\text { Range }\end{array}$ & $\begin{array}{c}\text { Polymorphonuclear } \\
\text { Median (per } \mathrm{mm}^{3} \text { ) } \\
\text { and Range }\end{array}$ & $\begin{array}{c}\text { Protein Median } \\
\text { (g/l) Normal } \\
0.15-0.45 \text { and } \\
\text { Range }\end{array}$ \\
\hline Myelitis & $38.5(0-265)$ & $0(0-300)$ & $2(0.14-2)$ \\
\hline Normal & $1(0-160)$ & $0(0-5)$ & $0.49(0.11-2.6)$ \\
\hline$P$ value & $.0053^{a}$ & .2082 & $.0017^{a}$ \\
\hline
\end{tabular}

${ }^{a}$ Indicates significance.
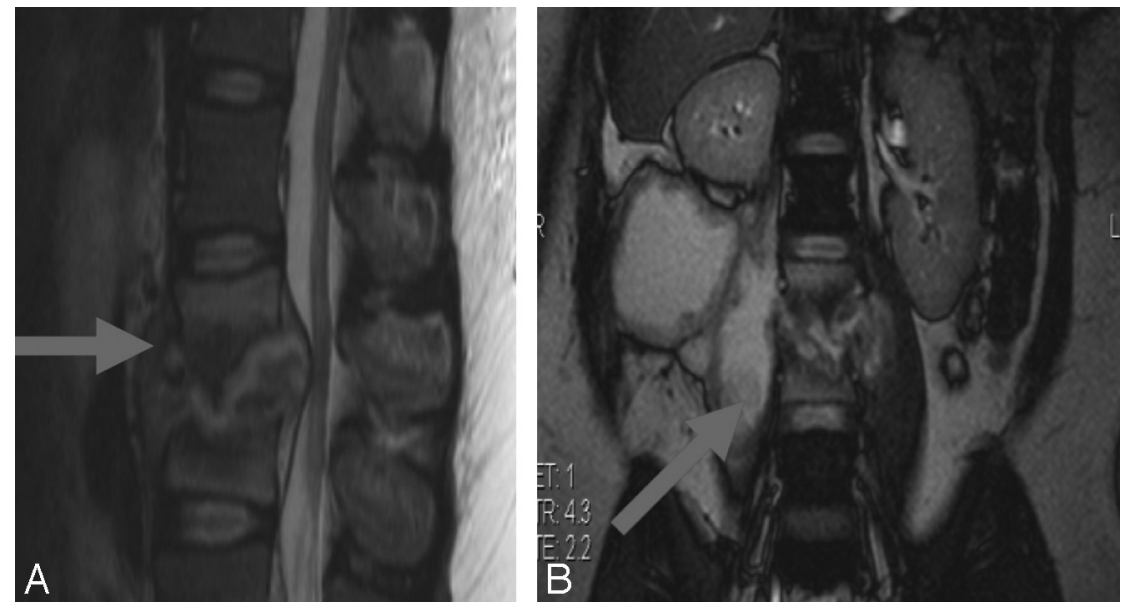

FIG 2. Tuberculous spondylo-diskitis. A, Sagittal T2-weighted MR imaging demonstrates caseous destruction of the L2-3 disk with epidural collection causing compression of the conus. B, Coronal FISP demonstrates large psoas abscesses communicating with the L2-3 disk. A thick walled collection displaces the right kidney superiorly.
$(42 \%)$ in the thoracic cord. Seven patients, 4 of whom were on ARVs, had discretely enhancing intramedullary lesions.

Arachnoiditis. In 25/64 cases (39\%), there was thickening, nodularity, and/or enhancement of the cauda equina. Discrete leguminous nodules with low signal on T2WI and solid enhancement postgadolinium were present in the CSF in 3 patients. Four had subtle enhancement of the pial surface of the cord or cauda equina roots. These findings were often in association with cord edema; however, in 8 patients, thickened cauda equina roots and increased signal in the CSF on precontrast T1-weighted imaging were isolated findings. Four patients in whom tuberculous meningitis had been diagnosed prior to their developing spinal symptoms had basal meningeal, intracerebral, and intramedullary ring enhancing lesions on MR imaging of the brain.

Epidural Abscess. In 13/64 patients (20\%), loculated and/or enhancing epidural/subarachnoid collections were associated with extensive cord signal abnormality (Fig 3 ).

\section{DISCUSSION}

Globally, TB is the most common cause of death among patients with AIDS, killing 1 in 3 patients. ${ }^{4}$

In South Africa, an estimated 5.7 million people are infected with HIV ( $11 \%$ of the population), making it the country with the highest prevalence of HIV in the world. ${ }^{5}$ A recent audit has shown a new TB index case load in excess of 1600 per 100,000 in the greater Cape Town area. ${ }^{2}$

The background high rate of $\mathrm{TB}$, together with the delay in the provision of antiretrovirals until late in the HIV epidemic, plausibly explains the large number of tuberculosis-related neurologic complications seen in the Western Cape. This study serves as the largest reported collection of HIV-positive patients presenting with myelopathy and is the first to categorize the MR imaging findings in a TB endemic area.

\section{Determining the Range of Causes of Myelopathy in Patients with HIV}

This study showed that two-thirds of HIV-positive patients presenting with myelopathy to our institution with a determined diagnosis have an infectious etiology. Mycobacterium tuberculosis is the most common infective agent in both spondylitic and nonspondylitic presentation, even in the setting of a normal MR imaging and often despite ARVs. Fifty-eight percent of the total group are women reflecting the heterosexual nature of transmission and sex pattern of HIV in South Africa.

\section{Other South African Experience}

In a study in 2001, of 33 HIV-positive South African patients presenting with myelopathy, Bhigjee et $\mathrm{al}^{6}$ found tuberculosis to be the most common etiologic agent and predicted that opportunistic infections would persist until routine antiretrovirals became widely available in South Africa. 


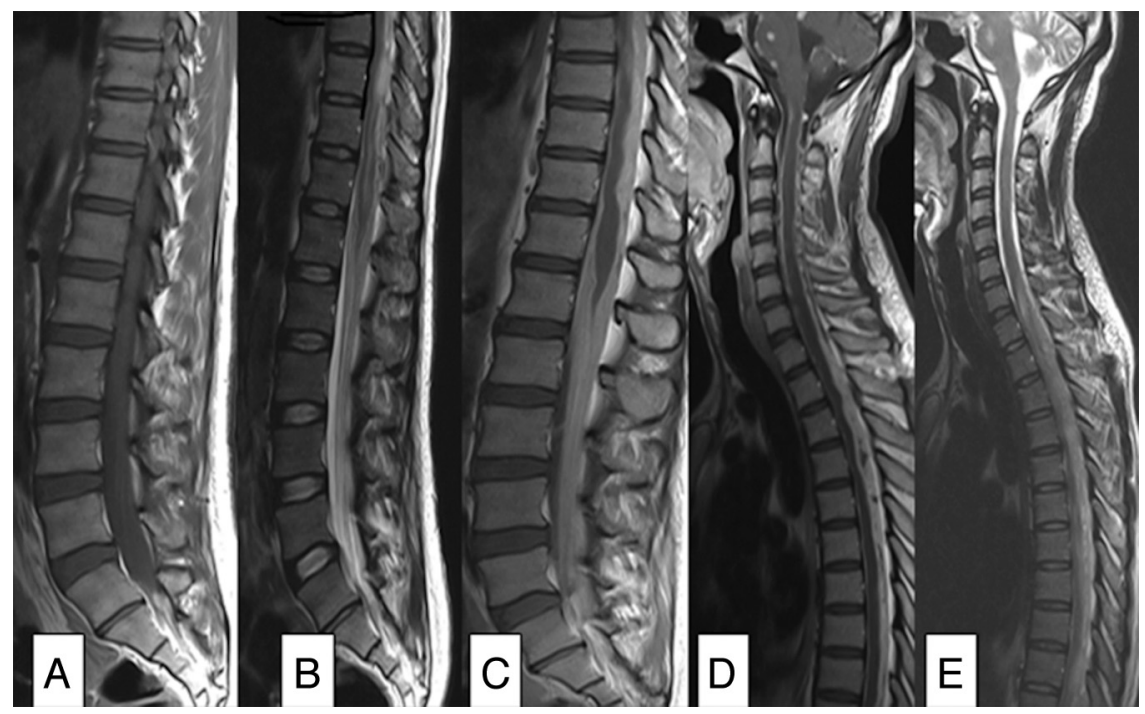

FIG 3. Nonspondylitic spinal tuberculosis. $A, T 1$ sagittal pregadolinium image with increased signal of the CSF. $B, T 2$ sagittal shows root thickening. $C$ and $D$, TT sagittal postgadolinium demonstrates circumferential cord and root enhancement. $E$, T2 sagittal shows loculated CSF with extensive cord signal abnormality.

Despite a nationwide roll-out of antiretroviral therapy, Modi et $\mathrm{al}^{7}$ found in a more recent study of 100 South African patients with nontraumatic myelopathy that infections were again largely responsible for myelopathy in the HIV-positive patients, with Mycobacterium tuberculosis being causative in 50\%. Varicella zoster, human T-lymphotropic virus 1 , and cytomegalovirus were implicated only rarely and incidental neoplastic and degenerative causes made up the remainder.

\section{The Developing World}

Studies of myelopathy in sub-Saharan Africa, India, Zimbabwe, Nigeria, and Ethiopia emphasize the role of infection as a cause of myelopathy in developing countries. Although none of these reports specifically examined an HIV cohort, HIV and TB were the most common infections described in patients presenting with myelopathy. ${ }^{8-10}$

\section{MR Imaging in HIV-Associated Myelopathy}

In a collaborative retrospective study published in 2000, Thurnher et $\mathrm{al}^{11}$ reviewed the MRI of $55 \mathrm{HIV}$-positive European and North American patients presenting with spine-related neurology. The study was limited by nonuniformity of clinical presentation and by the absence of a single imaging protocol (only 2 had the entire spine imaged). In 23 cases with osseous and epidural involvement, 4 were attributable to TB spondylodiskitis, 2 to pyogenic spondylodiskitis, and 7 to lymphoma.

In our study, $\mathrm{TB}$ spondylitis was diagnosed on MR imaging with a high degree of certainty with 57 of $65 \mathrm{MR}$ imaging-positive cases (88\%) being confirmed on open biopsy or by CSF. Localized epidural collection and kyphosis resulted in focal cord compression. None of these cases had diffuse cord swelling, signal abnormality, or arachnoiditis beyond the spondylitic segment.

Even in those in whom the disk appeared to be preferentially involved, pyogenic diskitis was not identified. Jung et $\mathrm{al}^{12}$ reported tuberculous spondylitis as having thin smooth enhancement of the abscess wall and a well-defined paraspinal abscess, whereas pyogenic spondylitis demonstrates a thick-walled, irregular collection centered on the disk. Given that the diagnosis in our institution is founded on biopsy, gadolinium is not administered routinely in this context.

Nonspondylitic tuberculous myelitis is less widely reported. Gupta et $\mathrm{al}^{13}$ described the MR imaging findings in a series of 20 patients with nonspondylitic intraspinal tuberculosis.

Our study suggests that in HIV, this "atypical" or nonosseous form of spinal tuberculosis is more common than previously described in TB endemic areas. ${ }^{14-17}$ The high prevalence of coexisting extra-spinal tuberculosis in our myelitis group argues strongly for a tuberculous etiology. The myelitis in these cases may be explained by granulomatous or caseous arachnoiditis with secondary vascular (arterial or venous) cord ischemia. The preponderance of thoracic cord signal abnormality in our patients would support this. In addition, our study demonstrates good correlation between inflammatory/ infective MR imaging features and inflammatory activity in CSF.

Other proposed mechanisms include edema surrounding hematogenous spread of tuberculomas directly to cord tissue ${ }^{18}$ or from the subarachnoid space ${ }^{19}$ and postinfectious or postinflammatory demyelination. Enhancing intramedullary lesions with surrounding edema are described in many conditions including tuberculosis, cryptococcosis, neurocysticercosis, toxoplasmosis, and schistosomiasis. Because cord biopsy is seldom an option, empiric treatment relies on knowledge of clinical status and CSF findings. HIV itself often results in mild CSF abnormalities. ${ }^{20}$ Our low CSF culture yield may reflect antituberculous treatment or the difficulty of culturing Mycobacterium tuberculosis from the CSF especially with HIV co-infection. ${ }^{21-24}$

Direct contiguous spread from infected cervical, mediastinal, or retroperitoneal lymph nodes may provide another route for spinal infection in the absence of bony involvement. This was convincingly demonstrated in only 2 of our cases.

\section{Nontuberculous Myelitis/Arachnoiditis}

Cytomegalovirus is usually characterized by painless radiculomyelitis and less commonly by necrotizing myelopathy. ${ }^{25}$ Commonly described with enhancement of the conus and thickened clumped roots, the diagnosis may be presumptive in the presence of enhancing roots on MR imaging and the presence of cytomegalovirus retinitis. ${ }^{11}$ This organism was found in the CSF of only 3 of our patients (2\%). One had coexisting disseminated tuberculous meningitis with floridly enhancing arachnoiditis, and 1 had only subtle CSF signal abnormality on T1WI but no root clumping. All 3 patients had profoundly reduced CD4 counts (less than 5 cells $/ \mu \mathrm{L}$ ). The absence of retinitis and the low prevalence on CSF 


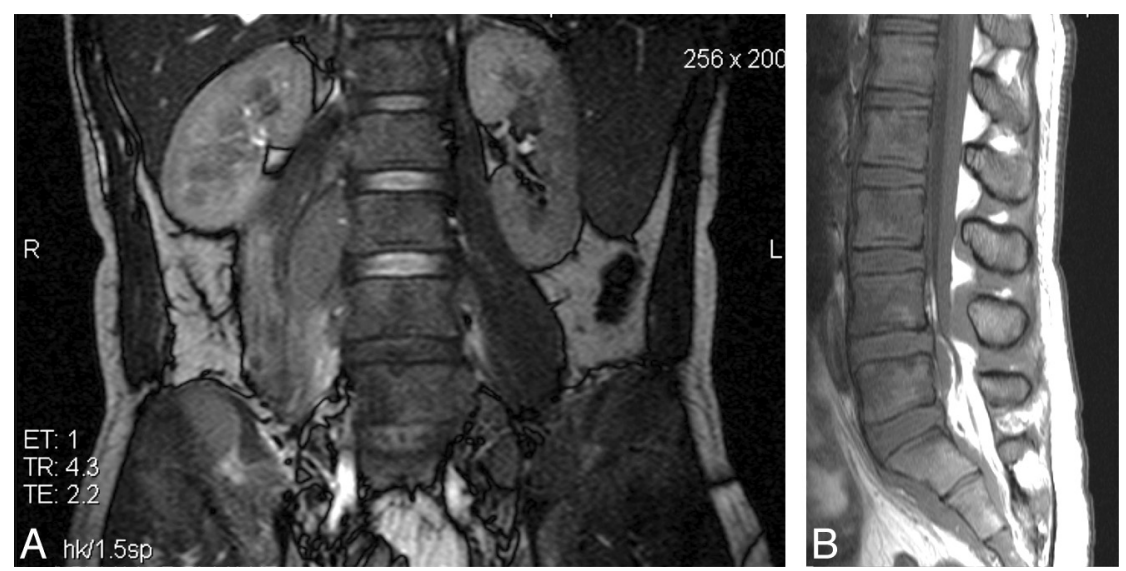

FIG 4. Burkitt lymphoma. A, Coronal FISP showing nonfluid-containing right $\mathrm{L} 2 / 3$ paraspinal and right pelvic masses infiltrating the right iliac bone and multiple low-signal lesions in both renal cortices. B, Sagittal T1 shows diffusely low marrow signal with isointense epidural masses posterior to the bodies of L5, S1, and S2.

specimens in both Bhigjee et $\mathrm{al}^{6}$ and our study suggests that cytomegalovirus may be a less common opportunistic infection in South African patients with AIDS than elsewhere.

Arterial and/or venous infarction may complicate meningovascular infection in CNS syphilis ${ }^{26}$; however, no cases of Treponema pallidum were documented in our study. Similar long segment cord involvement is seen on MR imaging in neuromyelitis optica. Explained by molecular mimicry, inflammation and damage to the spinal cord occur secondary to a shared antigenic profile between an invading virus, bacterium or plant protein, and spinal cord tissue. ${ }^{27}$ Aquaporin 4 antibody levels were not done routinely at the time of our study. Long segment cord signal abnormality is also described with $B_{12}$ deficiency, though no documented cases of pernicious anemia were encountered in our group.

Fungal causes of myelitis are extremely rare. Cryptococcal CNS involvement typically manifests as meningitis or parenchymal brain lesions. Spinal cord disease, when it occurs, may take the form of epidural abscess, chronic arachnoiditis, intramedullary granuloma, myelitis, or vasculitis with cord infarction. Although most cases are secondary to vertebral osteomyelitis, ${ }^{28}$ none of our spondylitis cases were attributable to Cryptococcus. Two had a normal MR imaging and a third with associated disseminated tuberculosis had florid arachnoiditis, cord edema, and splenic microabscesses. All 3 cases had very low CD4 counts.

Herpes zoster myelopathy is rare. This organism was isolated from the CSF of 3 patients, all of whom demonstrated long segment cord signal abnormality. Two had associated zoster skin rash at the time of presentation.

\section{Direct HIV Infection Resulting in Myelopathy}

HIV infection may cause an acute transverse myelitis on the basis of immune dysregulation even before the development of full-blown AIDS. During this early stage of infection, the myelopathy may be responsive to steroids and combination antiretroviral therapy. ${ }^{29}$

Vacuolar myelopathy frequently co-occurs with HIV-related encephalopathy and polyneuropathy and is a pathologic and postmortem diagnosis. ${ }^{25}$ The MR imaging findings include cord atrophy with or without signal abnormality and lack of enhancement. ${ }^{30}$ It is possible that vacuolar myelopathy explained some of our cases with bland CSF and negative MR imaging; however, postmortem results were available on only 1 of our patients whose death was attributed to subacute measles encephalopathy.

Diffuse infiltrative lymphocytosis syndrome warrants mention in the context of antigen-driven HIV manifestation. This condition represents a peripheral neuropathy rather than a myelopathy, may be symmetrical, acute, or subacute, but is always painful.

On MR imaging, there is typically leptomeningeal and cauda equina enhancement. Nerve biopsy is confirmatory with angiocentric CD8 infiltrates, prominent expression of HIV p24 protein in macrophages, and detection of the HIV genome by polymerase chain reaction in nerve homogenates. ${ }^{31}$ This diagnosis may have been overlooked in some of our patients.

\section{CONCLUSIONS}

Despite the limitations of a retrospective study and the lack of MR imaging contrast administration in all cases, our study re-emphasizes the importance of tuberculous infection in the development of acute myelopathy/cauda equina syndrome in the setting of HIV/AIDS. In the Western Cape, tuberculosis in its various forms accounts for at least $68 \%$ of cases with a documented etiology, often complicating tuberculosis at another site. Both spondylitis and myelitis/arachnoiditis may be diagnosed on MR imaging and may develop or progress on established anti-TB and ARV treatment.

MR imaging, in addition, allows for triage to medical or surgical treatment, though nonspondylitic infection lacks specificity on MR imaging even with the administration of gadolinium. Future research by using advanced physiologic MR imaging sequences (DWI and MR spectroscopy) and more accurate confirmatory testing with molecular-based assays of CSF may expand the role for MR imaging, both in diagnosis and management of this devastating disease presentation.

\section{APPENDIX 1}

\section{Definition of MR Imaging Classification}

- Spondylitis. Vertebral collapse; kyphosis; disk signal alteration/destruction/height loss; endplate destruction; paraspinal/ epidural mass. A confident diagnosis was reached when the paraspinal mass returned fluid signal and demonstrated subligamentous extension, or when there was endplate and disk involvement. Cord or thecal sac compression was considered present when there was loss of the anterior CSF signal at the level of kyphosis, indentation of the cord, or focal cord signal abnormality.

- Myelitis. Cord expansion/cord signal abnormality/enhancement within the thecal sac in the absence of spondylitic bone change.

- Arachnoiditis. Thickening/nodularity/or clumping of the cauda roots.

- Bone neoplasm. Expansion/collapse with epidural cord or AJNR Am J Neuroradiol 35:1634-41 Aug 2014 www.ajnr.org 

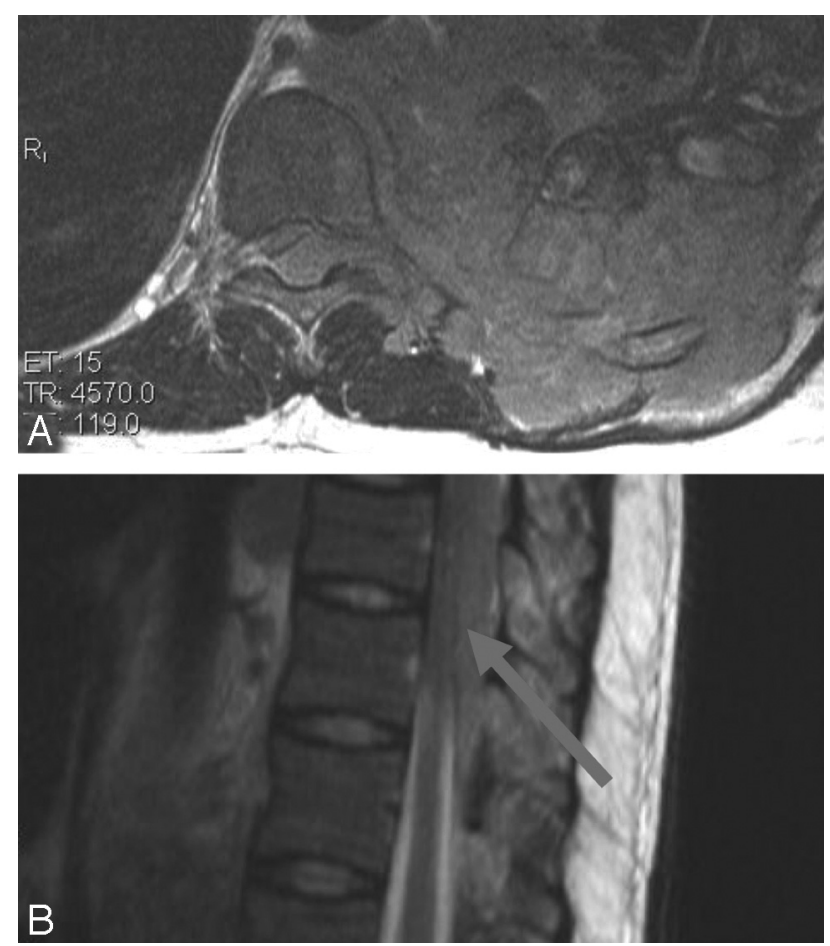

FIG 5. Plasmablastic lymphoma. A, Axial T2-weighted MR imaging shows a large destructive lobulated mass in the left hemithorax infiltrating the thoracic spinal canal via the neural foramen. B, T2weighted sagittal MR imaging showing posterior epidural cord compression. cauda compression/associated epidural thecal sac compression /“curtain” sign.

- Nonbone neoplasm. Intramedullary/extramedullary intrathecal/epidural soft tissue/contiguous spread from an adjacent chest wall or lung parenchymal, abdominal or pelvic mass, or lymphadenopathy suggesting lymphoma.

- Isolated syrinx. Cord expansion with nonenhancing intramedullary fluid signal (isointense to CSF on both T1 and T2WI) with or without loculations.

- Other. Disk herniation/degenerative spondolytic change.

\section{APPENDIX 2}

\section{Descriptions of MR Imaging Findings in Documented} Nontuberculous Infection

Varicella zoster was isolated from the CSF of 3 patients with features of myelitis on MR imaging, none of whom were on ARVs. A 31-yearold man with a relatively preserved CD4 (346 cells/ $\mu \mathrm{L})$ had been hospitalized for sepsis and a dermatomal skin rash typical for zoster 2 weeks before developing paraplegia and loss of sphincter control. His MR imaging demonstrated mild cord swelling and patchy leptomeningeal enhancement between T6 and T9. A second patient, a 49-yearold man with a CD4 of 158 cells/ $\mu \mathrm{L}$ presented with meningitis and myelopathy and had only patchy signal abnormality without cord expansion at T8/9. The third patient, a 28-year-old man (CD4 127 cells $/ \mu \mathrm{L}$ ) presented with a right $\mathrm{T} 6$ distribution zoster rash. His MR imaging demonstrated extensive cord swelling and signal abnormality between the midcervical level and the conus.

Cytomegalovirus was isolated from the CSF in 4 cases. The MR imaging was abnormal in only 2: one of these $(\mathrm{CD} 43$ cells $/ \mu \mathrm{L})$

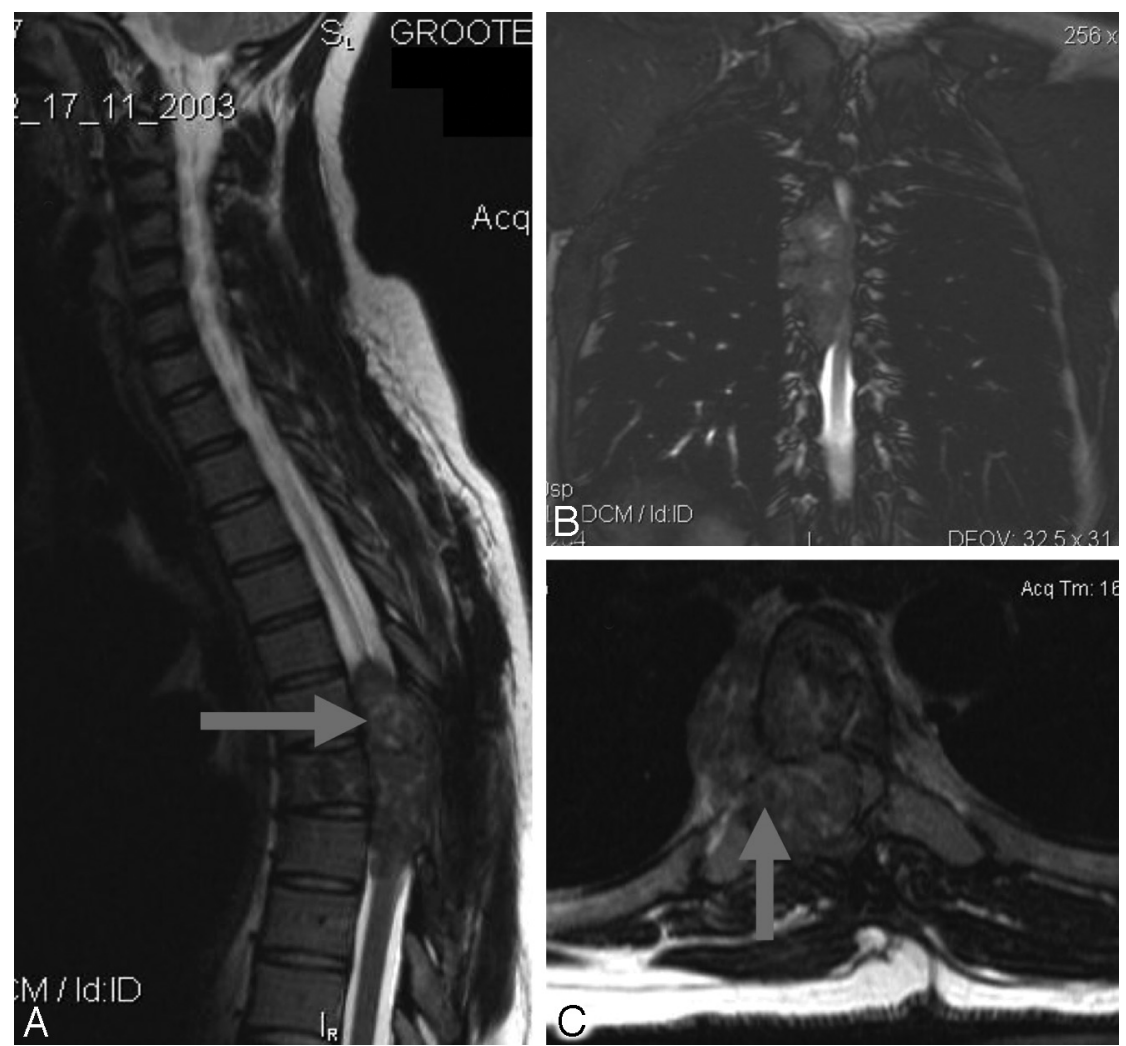

FIG 6. EB-associated myopericytoma. A, Sagittal T2-weighted MR imaging shows predominantly low-signal epidural mass causing significant cord compression at T6/7 with abnormal marrow in the adjacent vertebral body. $B$, Coronal FISP demonstrates improved conspicuity of marrow signal abnormality. C, Axial T2-weighted image shows tumor displacing and compressing the cord. edema between $\mathrm{C} 1$ and $\mathrm{C} 7$. The other had focal dural enhancement at T7/8 but no cord signal abnormality and no evidence of root enhancement.

Lymphoma was diagnosed histologically in 10 cases. Burkitts lymphoma was proved in 6 patients (Fig 4). Two patients had large B-cell and 2 had plasmablastic variant (Fig 5). Radiologically, there was no consistency in MR imaging appearance within histologic subtypes of lymphoma. These tumors were, however, distinct from the $\mathrm{TB}$ spondylitis group in that there was no endplate involvement, vertebral body height loss, or kyphosis. In 2 cases, the marrow signal was diffusely reduced throughout the spinal axis on T1 imaging. In several cases the cord or thecal sac was compressed by contiguous spread of paraspinal or mediastinal soft tissue into the epidural compartment via the neural foramina. Retroperitoneal and mediastinal lymphadenopathy was commonly identified on FISP imaging.

\section{EB/HIV Associated Tumors}

A 37-year-old man (CD4 47 cells/ $\mu \mathrm{L}$ ) presented with a T6 sensory level and demonstrated nonenhancing cord 
motor fallout. MR imaging revealed an enhancing dumbbellshaped mass expanding the right T4/5 neural foramen and causing epidural compression of the cord. The mass had low signal on T2WI, and histology revealed a spindle cell lesion with interlacing fascicles of smooth muscle fibers surrounding thick-walled vessels, consistent with an angioleiomyoma. The diagnosis was confirmed by a diffusely positive muscle-specific actin in the absence of positive staining for other immune-histochemical markers (EMA, S-100, Desmin, and CD34).

A 35-year-old woman (CD4 45cells/ $\mu \mathrm{L}$ ), presenting with motor weakness and a T6 sensory level, had an extradural soft tissue mass with low signal on T2WI and bony infiltration causing cord compression at T6/7. Histology revealed hypocellular and cellular areas. These were composed of relatively monomorphic oval to spindleshaped myoid appearing cells with multilayered concentric growth around vessels. The cells had eosinophilic and amphophilic (staining with both acid and basic dyes) cytoplasm. Cells were arranged in a storiform (cartwheel) pattern with scanty eosinophilic cytoplasm. Interspersed among the tumor cells were numerous small lymphocytes as well as more primitive cells with darker nuclei. Mitotic index and Ki67 were low and the tumor stained moderately positive for EB. IS100, epithelial membrane antigen (EMA), cytokeratin stains (AE1/ 3), B cell lymphoma 2 stain (BCL2), and haemopoetic progenitor cell stain (CD34) were negative. These findings favored an EB-associated smooth muscle tumor: myopericytoma (Fig 6).

\section{Non-HIV-Associated Cases}

Metastases and Primary Bone Tumors. Four patients had bony metastases. The MR imaging findings included posterior element/ pedicular infiltration, osseous destruction, and solid rather than fluid-containing paraspinal mass. Another feature favoring bone tumor over tuberculous spondylitis was the "curtain sign," referring to tumorous tissue bulging out laterally into the spinal canal on either side of the firmly attached midline posterior longitudinal ligament. In addition, 2 solitary plasmacytomas and a case of multiple myeloma were found.

\section{Disk Herniations}

Eight patients had large disk herniations causing cord or thecal sac compression: 3 cervical, 4 lumbar, and 1 at T10/11.

\section{ACKNOWLEDGMENTS}

We thank Dr N. Macingwane for help with identifying cases, and Prof Rodney Ehrlich and Dr Michele Youngleson for comments on earlier drafts.

\section{REFERENCES}

1. World TB Day, 24 March 2012. http://www.westerncape.gov.za/ news/world-tb-day-24-march-2012. Accessed April 18, 2014

2. Marais S, Pepper DJ, Schutz C, et al. Presentation and outcome of tuberculous meningitis in a high HIV prevalence setting. PloS One 2011;6:e20077

3. Jain AK. Tuberculosis of the spine: a fresh look at an old disease. J Bone Joint Surg Br 2010;92:905-13

4. World Health Organization. WHO report 2011. Global tuberculosis control. 2011. Available at: http://www.tbvi.eu/news-agenda/news/ news-message/who-2011-global-tuberculosis-control-report.html. Accessed August 20, 2013
5. Statistical release P0302. Mid-year population estimates 2013. http://beta2.statssa.gov.za/publications/P0302/P03022013.pdf. Accessed July 11, 2013

6. Bhigjee AI, Madurai S, Bill PL, et al. Spectrum of myelopathies in HIV seropositive South African patients. Neurology 2001;57:348-51

7. Modi G, Ranchhod J, Hari K, et al. Non-traumatic myelopathy at the Chris Hani Baragwanath Hospital. South Africa-the influence of HIV. QJM 2011;104:697-703

8. Zenebe G. Myelopathies in Ethiopia. East Afr Med J 1995;72:42-45

9. Owolabi LF, Ibrahim A, Samalia AA. Profile and outcome of nontraumatic paraplegia in Kano, northwestern Nigeria. Ann Afr Med 2011;10:86-90

10. Lekoubou Looti AZ, Kengne AP, Djientcheu Vde P, et al. Patterns of non-traumatic myelopathies in Yaounde (Cameroon): a hospital based study. J Neurol Neurosurg Psychiatry 2010;81:768-70

11. Thurnher MM, Post MJ, Jinkins JR. MRI of infections and neoplasms of the spine and spinal cord in 55 patients with AIDS. Neuroradiology 2000;42:551-63

12. Jung NY, Jee WH, Ha KY, et al. Discrimination of tuberculous spondylitis from pyogenic spondylitis on MRI. AJR 2004;182:1405-10

13. Gupta RK, Gupta S, Kumar S, et al. MRI in intraspinal tuberculosis. Neuroradiology 1994;36:39-43

14. Babhulkar SS, Tayade WB, Babhulkar SK. Atypical spinal tuberculosis. J Bone Joint Surg Br 1984;66:239-42

15. Pande KC, Babhulkar SS. Atypical spinal tuberculosis. Clin Orthop Relat Res 2002;May(398):67-74

16. Jena A, Banerji AK, Tripathi RP, et al. Demonstration of intramedullary tuberculomas by magnetic resonance imaging: a report of two cases. Br J Radiol 1991;64:555-57

17. Parmar $\mathrm{H}$, Shah J, Patkar D, et al. Intramedullary tuberculomas. $\mathbf{M R}$ findings in seven patients. Acta Radiol 2000;41:572-77

18. Trivedi R, Saksena S, Gupta RK. Magnetic resonance imaging in central nervous system tuberculosis. Indian J Radiol Imag 2009;19:256-65

19. Janse van Rensburg P, Andronikou S, van Toorn R, et al. Magnetic resonance imaging of miliary tuberculosis of the central nervous system in children with tuberculous meningitis. Pediat Radiol 2008; 38:1306-13

20. Price RW, Spudich S. Antiretroviral therapy and central nervous system HIV type 1 infection. J Infect Dis 2008;197(Suppl 3):S294-306

21. Croda MG, Vidal JE, Hernandez AV, et al. Tuberculous meningitis in HIV-infected patients in Brazil: clinical and laboratory characteristics and factors associated with mortality. Int J Infect Dis 2010;14:e586-91

22. Puccioni-Sohler M, Brandao CO. Factors associated to the positive cerebrospinal fluid culture in the tuberculous meningitis. Arq Neuropsiquiatr 2007;65:48-53

23. Berenguer J, Moreno S, Laguna F, et al. Tuberculous meningitis in patients infected with the human immunodeficiency virus. $N$ Engl J Med 1992;326:668-72

24. Marais S, Pepper DJ, Marais BJ, et al. V-associated tuberculous meningitis-diagnostic and therapeutic challenges. Tuberculosis (Edinb) 2010;90:367-74

25. Cho TA, Vaitkevicius H. Infectious myelopathies. Continuum (Minneap Minn) 2012;18:1351-73

26. Tsui EY, Ng SH, Chow L, et al. Syphilitic myelitis with diffuse spinal cord abnormality on MR imaging. Eur Radiol 2002;12:2973-76

27. Vaishnav RA, Liu R, Chapman J, et al. Aquaporin 4 molecular mimicry and implications for neuromyelitis optica. J Neuroimmunol 2013;260:92-98

28. Murthy JM. Fungal infections of the central nervous system: the clinical syndromes. Neurol India 2007;55:221-25

29. Hamada Y, Watanabe K, Aoki T, et al. Primary HIV infection with acute transverse myelitis. Intern Med 2011;50:1615-17

30. Chong J, Di Rocco A, Tagliati M, et al. MR findings in AIDS-associated myelopathy. AJNR Am J Neuroradiol 1999;20:1412-16

31. Moulignier A, Authier FJ, Baudrimont M, et al. Peripheral neuropathy in human immunodeficiency virus-infected patients with the diffuse infiltrative lymphocytosis syndrome. Ann Neurol 1997;41:438-45 